\title{
Acoustical role of ear canal in exposure to the typical occupational noise levels
}

\author{
Hadi Asady ${ }^{1,2 \ddagger}$, Adrian Fuente $^{3 \ddagger}$, Siamak Pourabdian $^{1}$, Farhad Forouharmajd ${ }^{1 * \mathbb{C}}$, Ismail Shokrolahi ${ }^{1}$ \\ Received: 14 Sep 2020 \\ Published: 5 May 2021
}

\section{Abstract}

Background: Exposure to noise associated with injuries has become a public health issue in recent years. This study aimed to show the role of the acoustical structure of the ear canal on the typical occupational sound pressure levels at different frequencies.

Methods: This cross-sectional study was done on 20-30-year-old participants. White noise was used at 3 levels, including 75, 85, and $95 \mathrm{~dB}$ as the stimulus sound pressure levels (SSPLs). The speakers had a 1.5-meter distance from the participants and at the height of 87 centimeters from the lab ground and were located in front of the participants. The SSPLs were measured outside (cavum part of the external ear) and inside the right ear of each participant. Measurements were done at the total sound pressure level and in the 1/1 octave frequencies. The duration for each measurement was 10 seconds. The independent sample $t$ test was used for the statistical analysis, and the equality of means were rejected at $\mathrm{p}<0.05$.

Results: There were $30(50 \%)$ males out of the 60 participants. The mean \pm SD for the age of all the participants was $23.29 \pm 2.93$ years. The total sound pressure level difference between the inside and outside of the ears of male and female participants was statistically significant $(\mathrm{p}<0.001)$ at the stimulus sound pressure levels. The peak resonance was observed in the frequencies $2000 \mathrm{~Hz}$ and $8000 \mathrm{~Hz}$ for males and $8000 \mathrm{~Hz}$ for females.

Conclusion: The ear canal can amplify the sounds and increase the sound pressure levels. This amplification was found to be greater for males than for females.

Keywords: Ear Canal, Resonance, Occupational Noise

Conflicts of Interest: None declared

Funding: We declare that this research received grant with No. 196162 from the Isfahan University of Medical Sciences.

*This work has been published under CC BY-NC-SA 1.0 license.

Copyright $₫$ Iran University of Medical Sciences

Cite this article as: Asady H, Fuente A, Pourabdian S, Forouharmajd F, Shokrolahi I. Acoustical role of ear canal in exposure to the typical occupational noise levels. Med J Islam Repub Iran. 2021 (5 May);35:58. https://doi.org/10.47176/mjiri.35.58

\section{Introduction}

Exposure to noise associated with the injuries has become a public health issue in recent years. In addition to having adverse impacts on the hearing system, noise may also lead to other $h$ armful health effects on the people,
Corresponding author: Dr Farhad Forouharmajd, forouhar@hlth.mui.ac.ir

‡ Hadi Asady and Adrian Fuente contributed equally to this work.

1. Department of Occupational Health Engineering, School of Health, Isfahan University of Medical Sciences, Isfahan, Iran

2. Social Determinants of Health Research Center, Saveh University of Medical Sciences, Saveh, Iran

3. École d'orthophonie et d'audiology, Faculty of Medicine, Université de Montréal, Montreal, Quebec, Canada

\section{$\uparrow$ What is "already known" in this topic:}

Different ways are used in our ears to the best possible impedance matching achieved, and somehow the impedance mismatch that is released. These ways are the greater area of the eardrum to the oval window, and amplification actions of the middle ear ossicle, the ear canal, shoulder, head, and pinna.

\section{$\rightarrow$ What this article adds:}

Based on our knowledge, these amplification mechanisms have been neglected in the occupational health-related noise measurements and the hearing protection programs; thus, in this study, the effects of the external auditory canal on the total sound pressure level and sound pressure levels at different frequencies were studied. Also, 3 SSPLs that are usual in the workplaces were used in this research. The ear canal can amplify the sounds and increase the sound pressure levels. This amplification is found to be greater in men than in women. The resonance ability of the ear canal is larger in some frequencies, especially in higher frequencies. This ability of the ear canal should be considered in the workplace noise evaluations. 
such as sleeping disturbance, impairment of cognitive performance in children, cardiovascular and pulmonary diseases, type 2 diabetes, adverse birth outcomes, and annoyance (1-3). The effects of noise on humans depend not only on the physical characteristics of the noise, including the sound pressure level and frequency content of noise, et cetera, but also on the physical, physiological, and psychological systems reactions (4). One of these systems is our ears. Different ways are used in our ears to the best possible impedance matching achieved, and somehow the impedance mismatch that is released. These ways include the greater area of the eardrum to the oval window (about 17:1), and amplification actions of the middle ear ossicle, the ear canal, shoulder, head, and pinna (5). Despite the fact that the impedance mismatch and sound amplification actions are crucial for our normal life, these actions may not be desirable in some circumstances and places. One of these circumstances is exposure to workplace noises. The noise level measurements are usually done using sound level meters (SLM) in the workers' environment in the occupational health. Finally, the judgments about the actions are done by considering the reference organization recommendations (OSHA PEL $=90$ $\mathrm{dBA}$; NIOSH REL = $85 \mathrm{dBA}$; and $\mathrm{ACGIH}=85 \mathrm{dBA}$ over the 8-hour period). According to the existing protocols about avoiding the workplace noise effects, some controlling actions, such as decreasing the working time or using earplugs or earmuffs, are considered due to the use of the recommended safe levels of the environmental sound pressure. However, with the existing amplification mechanisms in our ears, safe levels of environmental sound pressure level remain safe inside the ears. To our knowledge, these amplification mechanisms have been neglected in the occupational health-related noise measurements and the hearing protection programs.

The aim of this study was to quantify the effects of the external auditory canal on the total sound pressure level and sound pressure levels at different frequencies.

\section{Methods}

\section{Study Design}

This cross-sectional study was done in the sound and vibration laboratory of the Health Faculty of Isfahan University of Medical Sciences in 2019. White noise at 3 sound pressure levels (SPL), including 75, 85, and $95 \mathrm{~dB}$, were used as the stimulus sound pressure levels (SSPLs). For more precision, the sound pressure levels were checked before each measurement by a calibrated sound level meter (model TES-1357) close to each participant. The speakers that were located in front of the participants had a distance of 1.5 meters from them and a height 87 centimeters from the lab ground. The participants were asked not to change their head position and keep looking directly at the speakers during the tests. Labview software (V 2012), with the data acquisition card (DAQ) made by USA National Instrument Co (model MC-3642), was used for playing and recording the noises. The sound pressure levels were recorded outside (cavum part of the external ear) and inside (at $2.0 \mathrm{~cm}$ depths from the entrance to the ear canal) of the right ear of each participant at the fre- quencies of $63,125,250,500,1000,2000,4000$, and 8000 Hz. A circular-shaped microphone with a 2 millimeter diameter was used for this purpose. Calibration was done for the microphone. The time duration of 10 seconds (10 s) was considered for all the sound pressure level measurements. To minimize the probable effects of the confounding factors, all the participants in the study had the same environmental conditions during the measurements.

\section{Study Sample and Inclusion Criteria}

One of the inclusion criteria for the study was hearing health (not having infections and deformities of the hearing structure). This criterion was checked by a general physician before the study. No sensitivity and irritation to noise and the age range of 20-30 years were other inclusion criteria of the study. Sensitivity and irritation to noise were checked by a question from the participants, asking whether they were annoyed in their living environment by the following sounds: jingling of coins, dog barking, car engines, someone chewing, vacuum cleaner, or any other sounds. Eventually, 60 male and female students with negative responses were included in the study as participants using the simple random selection method. Written informed consent was obtained from all participants enrolled in the study.

\section{Statistical Analysis}

The data were analyzed using SPSS (V 26) software. Statistical analysis results were reported for the male and female participants separately. The mean and standard deviation (SD) were reported for the quantitative and qualitative variables, respectively. The independent sample $t$ test was used for the statistical analysis, and the equality of means was rejected at $\mathrm{p}<0.05$.

\section{Results}

Out of 60 participants, $30(50 \%)$ were men. The mean and standard deviation (mean $\pm \mathrm{SD}$ ) of the age of all participants was $23.29 \pm 2.93$ years. Also, the mean and standard deviation (mean $\pm \mathrm{SD}$ ) for height, weight, and BMI (body mass index) of all the participants were $171.1 \pm 10.45 \mathrm{~cm}, 65.93 \pm 15.1 \mathrm{~kg} / \mathrm{m}^{2}$, and $22.3 \pm 3.6$, respectively. The total sound pressure level difference between inside and outside of ear of men and women participants at the stimulus sound pressure levels of 75, 85, and $95 \mathrm{~dB}$ was statistically significant $(\mathrm{p}<0.001)$. Men showed higher differences compared to women at all 3 stimulus sound pressure levels. The resonance ability of the ear canals in both genders decreased with increasing the stimulus sound pressure levels (see more details in Table 1).

When the SSPL was $75 \mathrm{~dB}$ for men, the maximum mean difference of sound pressure level between inside and outside of the ears was seen at the frequency of 2000 $\mathrm{Hz}(\mathrm{MD}=6.6 \mathrm{~dB})$, while with increasing the SSPL, the maximum mean difference of the frequency also changed, and the maximum difference at the SSPL of $85 \& 95 \mathrm{~dB}$ was at the frequency of $8000 \mathrm{~Hz}(\mathrm{MD}=6.6 \& 4.5 \mathrm{~dB}$, respectively). 
Table 1. Mean, Standard Deviation, Mean Difference, and P Values of the Total Sound Pressure Level Inside and Outside of Participants' Ear

\begin{tabular}{|c|c|c|c|c|c|c|c|c|c|c|c|c|c|c|c|c|c|c|c|}
\hline & & \multicolumn{18}{|c|}{ Stimulus Sound Pressure Level (dB) } \\
\hline & & \multicolumn{6}{|c|}{75} & \multicolumn{6}{|c|}{85} & \multicolumn{6}{|c|}{95} \\
\hline & & Out: & & Insi & & $\mathrm{MD}^{\dagger}$ & $P^{\pi}$ & Outs & & Insi & & MD & $\mathrm{P}$ & Outs & & Insi & & MD & $\mathrm{P}$ \\
\hline \multirow{3}{*}{ Gender } & & Mean & $\mathrm{SD}^{\ddagger}$ & Mean & SD & & & Mean & SD & Mean & SD & & & Mean & SD & Mean & SD & & \\
\hline & Men & 74.9 & 1.2 & 80.3 & 2.7 & 5.4 & $<0.001$ & 85 & 1.2 & 1.2 & 1.2 & 4.1 & $<0.001$ & 94.8 & 1 & 96.5 & 0.6 & 1.7 & $<0.001$ \\
\hline & Women & 75 & 2.6 & 77.7 & 3.5 & 2.7 & 0.001 & 84.9 & 1.3 & 1.3 & 1.3 & 2.7 & $<0.001$ & 95.1 & 0.7 & 96 & 0.7 & 0.9 & $<0.001$ \\
\hline
\end{tabular}

\# Standard Deviation (SD); $\uparrow$ Mean difference $=$ inside-outside; $\uparrow$ Bold numbers $=$ significant $\mathrm{P}$ values.

Table 2. Mean, Standard Deviation, Mean Difference, and P values of the Sound Pressure Level Inside and Outside of Male Participants' Ear at Different Frequencies ( $\mathrm{n}=30$ )

\begin{tabular}{|c|c|c|c|c|c|c|c|c|c|c|c|c|c|c|c|c|c|c|c|}
\hline & & \multicolumn{18}{|c|}{ Stimulus Sound Pressure Level (dB) } \\
\hline & & \multicolumn{5}{|c|}{75} & \multicolumn{7}{|c|}{85} & \multicolumn{6}{|c|}{95} \\
\hline & & \multicolumn{2}{|c|}{ Outside } & \multicolumn{2}{|c|}{ Inside } & \multirow[t]{2}{*}{$\mathrm{MD}^{\dagger}$} & \multirow[t]{2}{*}{$P^{\mathbb{q}}$} & \multicolumn{2}{|c|}{ Outside } & \multicolumn{2}{|c|}{ Inside } & \multirow[t]{2}{*}{ MD } & \multirow[t]{2}{*}{$P$} & \multicolumn{2}{|c|}{ Outside } & \multicolumn{2}{|c|}{ Inside } & \multirow[t]{2}{*}{ MD } & \multirow[t]{2}{*}{$\mathrm{P}$} \\
\hline \multirow{9}{*}{ 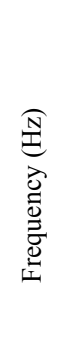 } & & Mean & $\mathrm{SD}^{\ddagger}$ & Mean & SD & & & Mean & SD & Mean & SD & & & Mean & SD & Mean & SD & & \\
\hline & 63 & 61.4 & 1.9 & 65.8 & 9.6 & 4.4 & 0.022 & 61.1 & 3.5 & 63.4 & 8.8 & 2.3 & 0.192 & 64.7 & 1.5 & 66.5 & 3.2 & 1.8 & 0.007 \\
\hline & 125 & 60.6 & 3 & 62 & 5 & 1.4 & 0.198 & 68.2 & 2.4 & 68 & 3.6 & -0.2 & 0.797 & 76.9 & 1.8 & 73.9 & 2.6 & -3 & $<0.001$ \\
\hline & 250 & 58.5 & 1.6 & 59 & 3.3 & 0.5 & 0.479 & 68.5 & 2.3 & 67.7 & 3 & -0.8 & 0.270 & 79.4 & 1.8 & 76 & 2.5 & -3.4 & $<0.001$ \\
\hline & 500 & 60.1 & 1.7 & 60.9 & 1.8 & 0.8 & 0.091 & 71 & 1.7 & 70.9 & 2.1 & -0.1 & 0.874 & 81.5 & 1.1 & 79.2 & 1.8 & -2.3 & $<0.001$ \\
\hline & 1000 & 69.3 & 1.6 & 70.2 & 1.3 & 0.9 & 0.016 & 79.8 & 1.6 & 80.6 & 1.6 & 0.8 & 0.084 & 89.9 & 1.3 & 87.8 & 2.1 & -2.1 & $<0.001$ \\
\hline & 2000 & 69.2 & 1.4 & 75.5 & 3 & 6.3 & $<0.001$ & 79.3 & 1.4 & 85.2 & 2.5 & 5.9 & $<0.001$ & 89 & 1.2 & 92.2 & 2.5 & 3.2 & $<0.001$ \\
\hline & 4000 & 68.9 & 2 & 73 & 4 & 4.1 & $<0.001$ & 79.5 & 2.2 & 82.6 & 4.3 & 3.1 & 0.001 & 89 & 1.8 & 90.8 & 2.5 & 1.8 & 0.003 \\
\hline & 8000 & 58.3 & 1.3 & 63.3 & 3.8 & 5 & $<0.001$ & 68.1 & 1.6 & 74.7 & 3.6 & 6.6 & $<0.001$ & 80.6 & 1.7 & 85.1 & 2.3 & 4.5 & $<0.001$ \\
\hline
\end{tabular}

¥ Standard deviation (SD); $\uparrow$ Mean difference $=$ inside-outside; $\uparrow$ Bold numbers $=$ significant $P$ values.

Table 3. Mean, Standard Deviation, Mean Difference, and P Values of the Sound Pressure Level Inside and Outside of Female Participants' Ear at Different Frequencies ( $\mathrm{n}=30$ )

\begin{tabular}{|c|c|c|c|c|c|c|c|c|c|c|c|c|c|c|c|c|c|c|c|}
\hline & & \multicolumn{18}{|c|}{ Stimulus Sound Pressure Level (dB) } \\
\hline \multirow{11}{*}{ 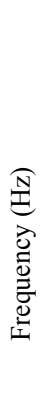 } & & \multicolumn{5}{|c|}{75} & & \multicolumn{6}{|c|}{85} & \multicolumn{5}{|c|}{95} & \\
\hline & & \multicolumn{2}{|c|}{ Outside } & \multicolumn{2}{|c|}{ Inside } & \multirow[t]{2}{*}{ MD } & \multirow[t]{2}{*}{$\mathrm{P}$} & \multicolumn{2}{|c|}{ Outside } & \multicolumn{2}{|c|}{ Inside } & \multirow[t]{2}{*}{ MD } & \multirow[t]{2}{*}{$\mathrm{P}$} & \multicolumn{2}{|c|}{ Outside } & \multicolumn{2}{|c|}{ Inside } & \multirow[t]{2}{*}{ MD } & \multirow[t]{2}{*}{$\mathrm{P}$} \\
\hline & & Mean & SD & Mean & SD & & & Mean & SD & Mean & SD & & & Mean & SD & Mean & SD & & \\
\hline & 63 & 61.2 & 3.6 & 58.9 & 8.2 & -2.3 & 0.175 & 60.3 & 1.6 & 58.7 & 7.8 & -1.6 & 0.270 & 65 & 1.7 & 65.6 & 3.7 & 0.6 & 0.446 \\
\hline & 125 & 62.1 & 3.8 & 61.4 & 4.7 & -0.7 & 0.491 & 68.8 & 1.4 & 67.6 & 3.2 & -1.2 & 0.068 & 78 & 1.2 & 75.8 & 2.6 & -2.2 & $<0.001$ \\
\hline & 250 & 60 & 3.2 & 58.6 & 3.8 & -1.4 & 0.142 & 69.2 & 1.5 & 67.8 & 2.7 & -1.4 & 0.020 & 80.2 & 1.6 & 78 & 2.4 & -2.2 & $<0.001$ \\
\hline & 500 & 61.7 & 3.8 & 60.1 & 3.9 & -1.6 & 0.127 & 71.7 & 1.2 & 70.8 & 2.2 & -0.9 & 0.046 & 82.5 & 1.1 & 80.4 & 1.6 & -2.1 & $<0.001$ \\
\hline & 1000 & 69.4 & 2.8 & 68.9 & 2.9 & -0.5 & 0.507 & 80.1 & 1.5 & 79.8 & 1.5 & -0.3 & 0.475 & 90.2 & 0.9 & 88.3 & 1.4 & -1.9 & $<0.001$ \\
\hline & 2000 & 68.2 & 2.9 & 72.4 & 3.7 & 4.2 & $<0.001$ & 78.9 & 1.4 & 82.6 & 2.6 & 3.7 & $<0.001$ & 88.6 & 0.8 & 90.7 & 1.8 & 2.1 & $<0.001$ \\
\hline & 4000 & 68.1 & 4.1 & 72.5 & 3.8 & 4.4 & $<0.001$ & 78.8 & 2.4 & 82.5 & 2.9 & 3.7 & $<0.001$ & 89 & 2.2 & 90.9 & 2 & 1.9 & 0.002 \\
\hline & 8000 & 58.3 & 4.1 & 66.2 & 4.2 & 7.9 & $<0.001$ & 69.3 & 2.5 & 76 & 2.8 & 6.7 & $<0.001$ & 81.1 & 2.3 & 86.2 & 1.9 & 5.1 & $<0.001$ \\
\hline
\end{tabular}


Also, the significant differences were mostly seen statistically for high frequencies at the 3 SSPL. However, the maximum mean difference of sound pressure levels between the inside and outside of the ears in women at all 3 SSPL was at the frequency of $8000 \mathrm{~Hz}(\mathrm{MD}=7.9$; 6.7; and $5.1 \mathrm{~dB}$, respectively). Also, the significant differences were mostly seen statistically for high frequencies at 3 SSPL (Tables 2 and 3).

\section{Discussion}

Our results showed that at the studied SSPLs, total sound pressure levels significantly increase inside the ear compared to the outside. In the previous studies in this regard, the researchers usually used 1 SSPL to study the ear canal behaviors, while the studied sound pressure levels were much under the routine occupational sound pressure levels (6-8). Therefore, 3 SSPLs that are usual in the workplaces were used in this research to study the ear canal behaviors. Since the previous studies have proved that ear morphology of men and women are different (911 ), and forasmuch as the presence of women at workplaces is increased nowadays (12), in this study, the responses of ear canals to the SSPLs were reported for the men and women separately. It was found that in all the 3 studied SSPLs, the elevation of the total sound pressure level inside the ear of men was higher than that of women. It seems that the ear canals of men can more increase the total sound pressure level than the women's ear canals. Despite the fact that the resonance ability of men's ears was more than that of women's, the ear resonance ability in both sexes was decreased with increasing the stimulus sound pressure level. Moreover, it seems that with increasing the exposure sound pressure level, not only the mean difference between the outside and inside of the ear canals in both sexes decreases, but also the women's ear canal behavior is mainly similar to men. Thus, it can be clearly seen that the ear canal amplification power, not only is related to the sound frequency, but also it depends on the sound pressure level. Since the total sound pressure level was not reported in the past related studies, we cannot compare our findings to others. According to the occupational health guidelines (related to the OSHA, $\mathrm{NIOSH}$, and ACGIH), the evaluation of noise level at workplaces was done by measuring the total sound pressure level in the centers of specific stations. Based on our findings, the ear canal amplifies the sound level in both genders. Therefore, in such conditions, exposure of individuals to the recommended safe levels of noise in workplaces may be dangerous and put the workers at the risk of high exposure. Also, since the male ear canal amplification ability is more than that of the females, male workers may be at more risk compared to the women workers. It seems that the different morphology of men's and women's ears, such as the larger volume of the men's ear canal compared to the women's $(13,14)$, makes the amplification ability different in both genders.

Since $1 / 1$ octave frequencies are widely used for the frequency analysis of noise at workplaces, in this study, the ear canal amplification behavior was determined in exposure to those frequencies. According to the results, sound pressure level amplification was not observed at different SSPLs in all the studied frequencies. At the SSPL of $75 \mathrm{~dB}$ and in all the measured frequencies, inside sound pressure levels in the men's ear were higher than that of the outside. Sound pressure amplification ability of men's ear canal for the frequency range of $125-1000 \mathrm{~Hz}$ was less than lower and higher frequencies, and the amplification peak was observed at the frequency of $2000 \mathrm{~Hz}$. These findings were consistent with the findings by Liu et al (7). Sound pressure resonance was not observed for frequencies of less than $1000 \mathrm{~Hz}$ in the women's ear canals and also the resonance peak was at the frequency of $8000 \mathrm{~Hz}$ in them. These findings were consistent with Hellstrom and Santos observations $(15,16)$. Since the ear canal volume in women is smaller than that of men, we expected that the peak of amplification would shift somewhat to higher frequencies in women.

At the SSPL of $85 \mathrm{~dB}$ and in some frequencies, including 63 and greater than $500 \mathrm{~Hz}$, the inside sound pressure levels in the men's ear were higher than that in the outside. Also, the peak amplification was observed at the frequency of $8000 \mathrm{~Hz}$. The same pattern was approximately seen in the women's ear canal. It seems that with increasing the SSPL, the frequency of resonance peak shifts to the higher frequencies in the men's ear canal but stays unchanged in women. Moreover, with increasing the SSPL, the ear canal amplification in men is lost at the lower frequencies $(<1000 \mathrm{~Hz})$. It seems that with increasing the exposure sound pressure levels, the men's ear canals cannot amplify the sound pressure levels at lower frequencies. At the SSPL of $95 \mathrm{~dB}$, the men's and women's ear canals are more likely to respond to the SSPL. At this value of SSPL, significant resonance is also revealed at the low frequencies. It seems that the ear canal can resonate at the low frequencies in the higher sound pressure levels. Since the accepted time weighted average (TWA) for an 8-hour exposure to workplace noise in some countries is $85 \mathrm{~dB}$, the resonance ability of the ear canal should be considered at the occupational hearing protection programs.

We could not measure the sound pressure levels of both ears of the participants. Different morphology of the right and left ears was proved by the previous studies $(17,18)$. Thus, it is recommended to further assess the ear canals of both ears at the wider range of SSPL in future studies.

\section{Conclusion}

The ear canal can amplify the sounds and increase the sound pressure levels. This amplification is found to be greater in men than in women. Resonance ability of the ear canal is larger in some frequencies, especially in the higher rates. This ability of the ear canal should be considered in the workplace noise evaluations. In such situations, the workers may be exposed to unaccepted noise levels in the workplaces, even under the occupational safe levels because of the resonance ability of the ear canal in both genders.

\section{Acknowledegement}

The authors would like to thank all students who partic- 
ipated in this study.

\section{Conflict of Interests}

The authors declare that they have no competing interests.

\section{References}

1.Li XT, Dong Q, Wang BS, Song HY, Wang SZ, Zhu BL. The Influence of Occupational Noise Exposure on Cardiovascular and Hearing Conditions among Industrial Workers. Sci Rep. 2019;9:7.

2. Hosseinabadi MB, Khanjani N, Ebrahimi MH, Mirbadie SR, Biganeh $\mathrm{J}$. The effects of industrial noise exposure on lipid peroxidation and antioxidant enzymes among workers. Int Arch Occup Environ Health. 2019;92(7):1041-6.

3. Basner M, Babisch W, Davis A, Brink M, Clark C, Janssen S, et al. Auditory and non-auditory effects of noise on health. Lancet. 2014;383(9925):1325-32.

4. Salmon V. The nature of noise. Water, Air, and Soil Pollution. 1973;2(3):257-65.

5. Kim YH. Sound propagation: an impedance based approach: John Wiley \& Sons; 2010. 86 p.

6. Silva APRd, Blasca WQ, Lauris JRP, Oliveira JRMd. Correlation between the characteristics of resonance and aging of the external ear. Codas. 2014;26:112-6.

7. Liu JL, Qin XL, Wang LH, Liang CY, Jiang T. Measurement of sound pressure level at outlet of external auditory canal and eardrum. Zhonghua Lao Dong Wei Sheng Zhi Ye Bing Za Zhi. 2003;21(5):3535 .

8. Chen S, Deng J, Bian L, Li G, editors. A new method to estimate sound energy entering the middle ear. 2013 35th Annual International Conference of the IEEE Engineering in Medicine and Biology Society (EMBC); 2013 3-7 July 2013.

9. Brucker MJ, Patel J, Sullivan PK. A morphometric study of the external ear: Age- and sex-related differences. Plast Reconstr Surg. 2003;112(2):647-52.

10. Bozkir MG, Karakas P, Yavuz M, Dere F. Morphometry of the external ear in our adult population. Aesthetic Plast Surg. 2006;30(1):81-5.

11. Sforza C, Grandi G, Binelli M, Tommasi DG, Rosati R, Ferrario VF. Age- and sex-related changes in the normal human ear. Forensic Sci. Int. 2009;187(1-3):110.e1-7.

12. Powell A, Mor F. Women and the economy. report. 2019 Mar. Report No.: CBP06838.

13. Grewe J, Thiele C, Mojallal H, Raab P, Sankowsky-Rothe T, Lenarz $T$, et al. New HRCT-based measurement of the human outer ear canal as a basis for acoustical methods. Am J Audiol. 2013;22(1):65-73.

14. Manchaiah V, Durisala N, Marimuthu V. Tympanometric profiles for Chinese older adults. Audiol Res. 2017;7(2):190-.

15. Hellström PA. The relationship between sound transfer functions and hearing levels. Hear Res. 1995;88(1-2):54-60.

16. dos Santos I, Colella-Santos MF, do Couto CM. Sound pressure level generated by individual portable sound equipment. Braz J Otorhinolaryngol. 2014;80(1):41-7.

17. Barnes J, Sabo RT, Coelho DH. A novel method to measure the external auditory canal: Normative data and practical implications. Am J Otolaryngol. 2018;39(2):146-9.

18. Osunwoke E, Vidona W, Atulegwu G. Anthropometric study on the anatomical variation of the external ear amongst Port Harcourt students. Int J Anat Var. 2018;11(4):144. 